\title{
Origin of Hysteresis Observed During Fatigue of Ceramic-Matrix Composites
}

\author{
Temel Kotil, John W. Holmes* and Maria Comninou \\ Ceramic Composites Research Laboratory, Department of Mechanical Engineering and Applied Mechanics, \\ The University of Michigan, Ann Arbor, Michigan 48109-2125
}

Possible mechanisms for the hysteresis in stress-strain response observed during fatigue of fiber-reinforced ceramics are examined analytically. In the model developed, the microstructure of a unidirectional composite is divided into adjacent cells, each containing a single fiber. The compliance of each cell is modeled by a series of springs, with frictional sliding of fibers represented by sliding blocks. Fatigue damage is modeled by allowing fibers to debond and fracture on a random cycle-by-cycle basis. The magnitude of the interfacial shear between the fibers and matrix is shown to play a significant role in determining the extent of hysteresis observed during fatigue loading of unidirectional composites. Practical considerations, such as the influence of fiber volume fraction on macroscopic fatigue behavior, are also discussed. [Key words: composites, fatigue, hysteresis, shear, silicon nitride.]

\section{Introduction}

$\mathbf{F}$ IBER-REINFORCED ceramic-matrix composites are under development for use in elevated-temperature applications. Many of the potential applications of these composites will involve fatigue loading (e.g., gas turbine rotors, vanes, and combustors). Although the mechanisms of toughening in fiberreinforced ceramics have been widely studied, ${ }^{1-8}$ little information is available concerning the relationship between composite microstructure and macroscopic fatigue behavior. In cyclic loading experiments at room temperature, Marshall and Evans ${ }^{9}$ have noted hysteresis in the tensile stress-strain response of fiber-reinforced ceramics; this hysteresis has been attributed to the interfacial friction that exists between the fiber and matrix. Similar hysteresis in stress-strain response has also been observed by Marshall and Oliver ${ }^{10}$ during loading and unloading of individual fibers embedded in a ceramic matrix.

In long-term tensile fatigue testing of laminated $0^{\circ}$ and $0^{\circ} / 90^{\circ} \mathrm{SiC}$-fiber/aluminosilicate-matrix composites, Prewo et al. ${ }^{11,12}$ have found considerable hysteresis in the tensile fatigue response of these materials. Holmes et al. ${ }^{13}$ also observed pronounced hysteresis and strain ratchetting during elevated-temperature tensile fatigue testing of a unidirectional SiC-fiber $/ \mathrm{Si}_{3} \mathrm{~N}_{4}$-matrix composite (hereafter denoted as $\mathrm{HP}-\mathrm{SiC}_{f} / \mathrm{Si}_{3} \mathrm{~N}_{4}$ ). Hysteresis in the cyclic stress-strain response of fiber-reinforced ceramics typically occurs when the maximum fatigue stress exceeds the monotonic proportional limit of the composite $;^{11-13}$ the current work examines possible mechanisms for this hysteresis.

A. G. Evans - contributing editor

Manuscript No. 198216. Received July 28, 1989; approved February 23,1990 .

*Member, American Ceramic Society.

\section{Analysis and Discussion}

\section{(1) Sliding-Friction Model for Stress-Strain Hysteresis}

Based upon SEM observations of fatigue damage in unidirectional $\mathrm{HP}-\mathrm{SiC}_{f} / \mathrm{Si}_{3} \mathrm{~N}_{4}$ composites, ${ }^{13}$ the microstructural damage present at an intermediate stage of fatigue is modeled as a series of fatigue-damaged zones surrounded by undamaged zones (Fig. 1). It is assumed that five fatiguedamaged zones are present in a gage length of $30 \mathrm{~mm}$. (From SEM observations, the number of fatigue-damaged zones observed within a $30-\mathrm{mm}$ gage length was typically between two and seven.) Each fatigue-damaged zone contains both fractured and unfractured fibers that partially bridge a matrix crack (Fig. 2(a)).

During tensile fatigue loading, the crack-opening displacement of a matrix crack ( $L_{0}$ in Fig. 2(a)) has contributions from partial fiber pullout and elastic deformation of the fibers that bridge the crack. A residual stress $\sigma_{r}$ acts normal to the axis of all fibers (Fig. 2(b)). In general, $\sigma_{r}$ has contributions from the transverse thermal-expansion mismatch and Poisson's ratio mismatch between the fibers and the matrix. For a given temperature, the contribution to $\sigma_{r}$ from the thermalexpansion mismatch would be constant (independent of the applied load); however, the contribution arising from a mismatch in Poisson's ratio between the fiber and matrix would depend upon the instantaneous level of the fatigue load. As a simplification, $\sigma_{r}$ is assumed constant in the analysis that is presented here. After initial debonding and fiber fracture, the residual stress acting on a fiber gives rise to an interfacial shear $\tau_{i}=\sigma_{r} \mu$ ( $\mu$ is the interfacial friction coefficient) that resists sliding of the fiber within the matrix during loading and unloading. As with $\sigma_{r}$, the interfacial friction coefficient is assigned a constant value in the analysis (this simplification is discussed later). For a fiber of diameter $d_{f}$, the friction force $\left(F_{\text {fric }}\right)$ acting on the current embedded length of a fiber $\left(L_{e}\right)$ is given by

$$
F_{\text {fric }}=\pi \tau_{i} L_{e} d_{f}
$$

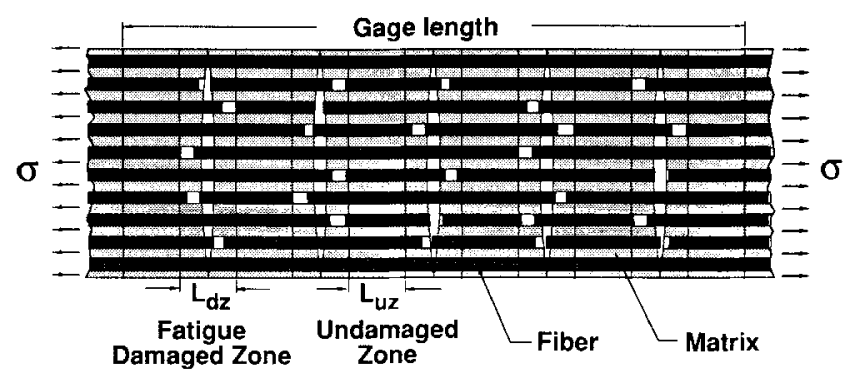

Fig. 1. Schematic representation of fatigue damage in a unidirectional fiber-reinforced/ceramic-matrix composite. The gage section of the composite is separated into a series of fatigue-damaged and undamaged zones. The model shown corresponds to the latter stages of fatigue damage, after a significant amount of fiber fracture has occurred. 


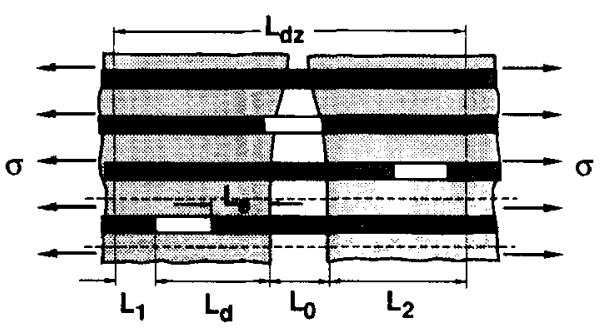

(a)

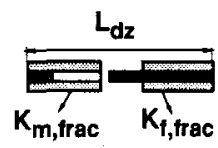

(b)

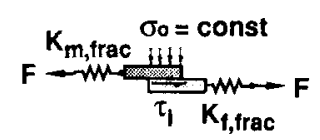

(c)
Fig. 2. (a) Schematic representation of a single fatigue-damaged zone. The matrix crack is partially bridged by fibers. (b) $K_{\text {f, frac }}$ and $K_{m \text { frac }}$ represent the stiffness of the unit cell in a fatigue-damaged zone (shown with dotted lines in (a)). (c) After debonding, a residual stress $\sigma_{r}$ produces an interfacial shear $\tau_{i}=\sigma_{r} \mu$, which acts over the current embedded length of the fiber $L_{e} . L_{1}$ and $L_{2}$ represent the fiber segments which remain bonded within a given fatigue-damaged zone.

Although the interfacial friction coefficient and residual stress are assumed constant, the magnitude of $F_{\text {fric }}$ decreases as fibers pull out of the matrix (decreasing $L_{e}$ ), and increases during unloading as fibers slide back into the matrix (increasing $L_{e}$ ).

The total length over which fatigue damage occurs (e.g., the gage section of a specimen) is further divided into parallel cells that span both the fatigue-damaged zones and undamaged zones. Each cell contains either a fractured or unfractured fiber, and the surrounding matrix (Fig. 3). The overall compliance of a cell depends upon whether it contains a fractured or unfractured fiber and upon the debond length assigned to each of the fractured fibers. For cells containing a fractured fiber, it is assumed that the fiber fractures once within each of the five fatigue-damaged zones. Moreover, for simplicity, each cell containing a fractured fiber is assigned the same debond length, $L_{d}$, for all fatigue-damaged zones spanned by a given cell.

For cells containing a fractured fiber, the stiffness contribution $\left(K_{m, \text { frac }}\right)$ of the matrix and associated fiber segment $L$, (see Fig. 2) within a fatigue-damaged zone is given in terms of the tensile modulus of the matrix, $E_{m}$, the cross-sectional

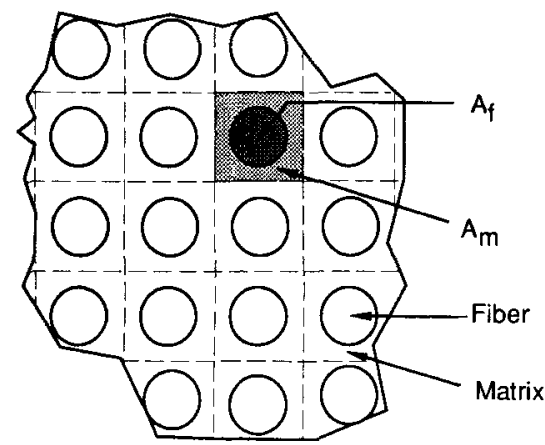

Fig. 3. Idealized cross section of composite. The composite is divided into square cells containing one fiber and the surrounding matrix. The cells are physically isolated from one another. $A_{f}$ and $A_{m}$ are the cross-sectional area of the fiber and surrounding matrix within each cell, respectively.

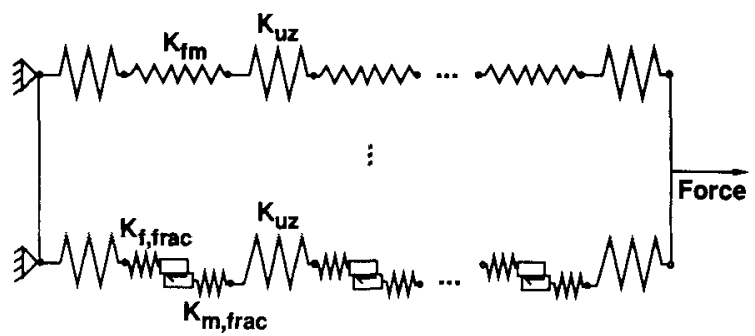

Fig. 4. Schematic representation of the variation in compliance along individual cells which span the gage section. Each series of springs represents one cell. The contribution to stiffness from unfractured fibers and the surrounding matrix within the fatigue-damaged zones, as given by $K_{f m}$, $K_{f, f r a c}$, and $K_{m, f r a c}$, represent the stiffnesses of fractured fibers and surrounding matrix within these zones. $K_{u z}$ represents the stiffness of the undamaged regions.

area of the matrix surrounding the fiber, $A_{m}$ (Fig. 3), and the fiber debond length $L_{d}$ :

$$
K_{m, f r a c}=\frac{(\beta+1) E_{m} A_{m}}{(\beta+1) L_{d}+L_{1}}
$$

where $\beta$ is defined in terms of the fiber modulus $E_{f}$ and fiber cross section $A_{f}$ :

$$
\beta=\frac{E_{f} A_{f}}{E_{m} A_{m}}
$$

The contribution to cell stiffness from a fractured fiber and surrounding matrix within a fatigue-damaged zone, $K_{f, \text { fract }}$, is calculated in a similar fashion:

$$
K_{f, f r a c}=\frac{(\beta+1) E_{f} A_{f}}{(\beta+1) L_{d}+\beta L_{2}}
$$

For cells containing unfractured fibers, the effective cell stiffness, $K_{f m}$, within a fatigue-damaged zone of length $L_{d z}$ is given by

$$
K_{f m}=\frac{E_{f} A_{f}+E_{m} A_{m}}{L_{d z}}
$$

The effective stiffness, $K_{u z}$, of the undamaged zones (surrounding each fatigue-damaged zone) is given in terms of the length of the undamaged zones, $L_{u z}$ :

$$
K_{u z}=\frac{E_{f} A_{f}+E_{m} A_{m}}{L_{u z}}
$$

A schematic representation showing the manner in which the compliance of each cell changes along its length is given in Fig. 4.

\section{(2) Application of Model}

When performing the calculations for stress-strain response, it is assumed that a matrix crack forms within each fatigue-damaged zone on the first loading cycle, without debonding of fibers. The fibers are next allowed to debond and fracture on a cycle-by-cycle basis, with a debond length $L_{d}$ assigned to each fiber by a random number generator. Physically, the cycle-by-cycle fracture of fibers could be caused by differing statistical strengths or, at elevated temperature, by differing oxidation rates of the fiber and fiber/ matrix interface.

The model will first be used to predict the magnitude of the hysteresis that would be expected for the experimental conditions employed in earlier elevated-temperature fatigue testing of unidirectional HP-SiC $f / \mathrm{Si}_{3} \mathrm{~N}_{4}$ composites with 30 vol\% of fibers. ${ }^{13}$ Required input data for the model are the fiber debond length $L_{d}$, the tensile modulus of the fiber and matrix, and frictional shear strength $\tau_{i}$ (i.e., the interfacial shear present after debonding of a fiber). For a maximum 
fatigue stress of $220 \mathrm{MPa}(10 \%$ above the monotonic proportional limit), $L_{d}$, measured in the SEM after fatigue failure of the $\mathrm{HP}-\mathrm{SiC}_{f} / \mathrm{Si}_{3} \mathrm{~N}_{4}$ composites, ranged from 0 to $2200 \mu \mathrm{m}$ (Fig. 5). The large variation in debond length is attributed to the random location of defects along the fiber. The frictional shear strength for this system has not been measured. However, for those systems where data is available (e.g., SCS-6 fibers in a glass matrix), the frictional shear strength measured at room temperature typically ranges from 0 to $30 \mathrm{MPa},{ }^{15-18}$ with the majority of the data clustered near 1 to $5 \mathrm{MPa}$. (For a comprehensive discussion of interfacial shear strength measurements the reader is referred to Kerans et al. ${ }^{19}$ ) In the calculations, the frictional shear strength was set at $4.5 \mathrm{MPa}$; the tensile modulus of the SCS-6 fibers at $350 \mathrm{GPa}$; and the modulus of the hot-pressed $\mathrm{Si}_{3} \mathrm{~N}_{4}$ matrix at $300 \mathrm{GPa}$. (As discussed in detail below, the frictional shear strength has a strong influence on the amount of hysteresis predicted.)

The predicted cyclic stress-strain response of the composite is given in Fig. 6 for $0 \%, 20 \%, 40 \%, 60 \%$, and $75 \%$ fiber fractures. (After $75 \%$ of the fibers have fractured, the stress carried by the remaining fibers would approach $\approx 85 \%$ of the fracture strength of the SCS $-6 \mathrm{SiC}$ fibers.) Comparing Figs. 6 and 7, the model adequately predicts the magnitude of hysteresis and strain ratchetting observed during fatigue testing but underestimates the increase in compliance. Although the model does predict an initial decrease in stiffness during loading, it does not predict the slight increase in stiffness that has been experimentally observed as the peak fatigue stress was approached (Fig. 7). The "S"-shaped hysteresis loops that were found experimentally are attributed to an increase in interfacial friction as the loading rate approaches zero (near stress reversal).

\section{(3) Influence of Frictional Shear Strength on Predicted} Fatigue Behavior

For the composite ( $\mathrm{HP}-\mathrm{SiC}_{f} / \mathrm{Si}_{3} \mathrm{~N}_{4}$ ) and loading conditions described above, the influence of frictional shear strength on

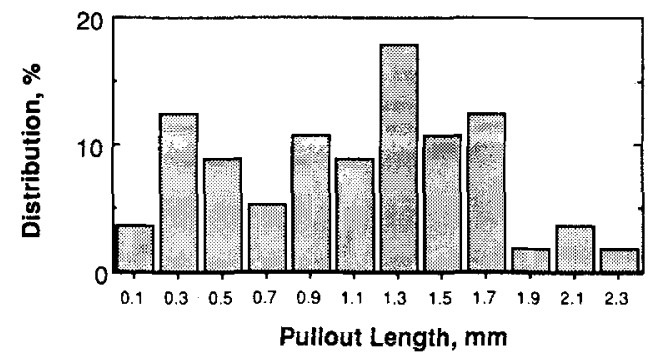

Fig. 5. Distribution of fiber pullout lengths observed after fatigue failure of hot-pressed $\mathrm{SiC}_{f} / \mathrm{Si}_{3} \mathrm{~N}_{4}$ composites.

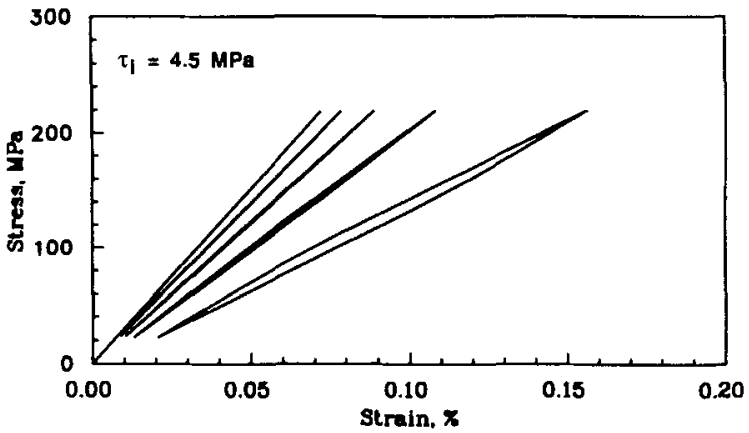

Fig. 6. Predicted hysteresis in the cyclic stress-strain response of hot-pressed $\mathrm{SiC}_{f} / \mathrm{Si}_{3} \mathrm{~N}_{4}$. The calculations assume the loading conditions given in Fig. 7. From left to right, the curves correspond to fracture of $0 \%, 20 \%, 40 \%, 60 \%$, and $75 \%$ of all fibers.

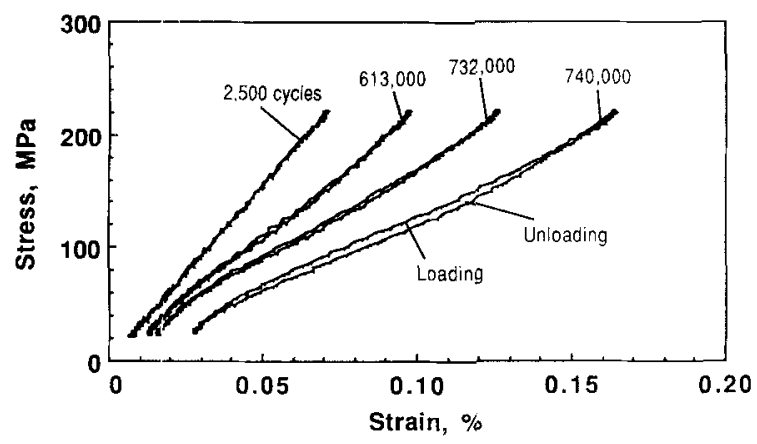

Fig. 7. Elevated temperature $\left(1000^{\circ} \mathrm{C}\right)$ cyclic stress-strain behavior of hot-pressed $\mathrm{SiC}_{f} / \mathrm{Si}_{3} \mathrm{~N}_{4}$ at a maximum fatigue stress $10 \%$ above the monotonic proportional limit of the composite. The fatigue testing was conducted at a sinusoidal frequency of $10 \mathrm{~Hz}$ and a stress ratio $\left(\sigma_{\min } / \sigma_{\max }\right)$ of 0.1 .

the predicted cyclic stress-strain behavior of the composite has been examined. The following three cases are considered: zero frictional shear strength, moderate frictional shear strength, and high frictional shear strength.

(A) Zero Frictional Shear Strength: As shown in Fig. 8(a), hysteresis or strain ratchetting is not predicted for zero friction shear stress. Physically, because the fractured fibers are free to slide within the matrix, zero frictional energy dissipation will occur during fatigue loading. The increase in compliance is due to the cycle-by-cycle fracture of fibers.

(B) Moderate Frictional Shear Strength: At a frictional shear strength of $0.5 \mathrm{MPa}$, both hysteresis and strain ratchetting begin to appear (Fig. 8(b)). Hysteresis becomes a maxi-

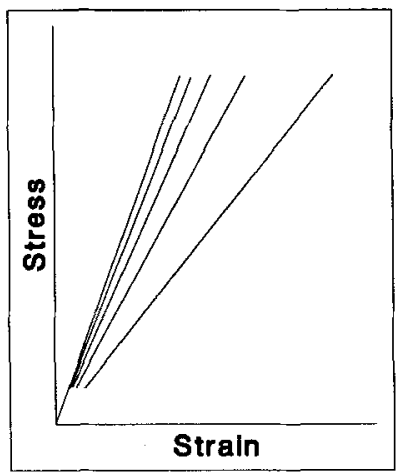

(a) $\tau_{i}=0 \mathrm{MPa}$

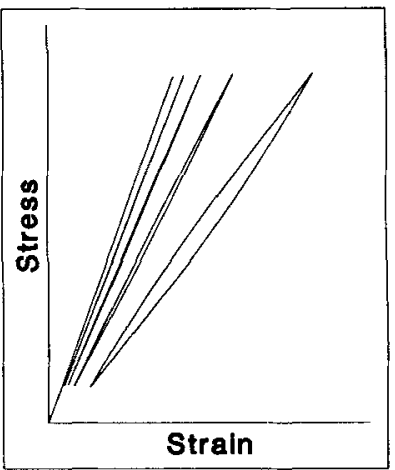

(c) $\tau_{i}=2.5 \mathrm{MPa}$

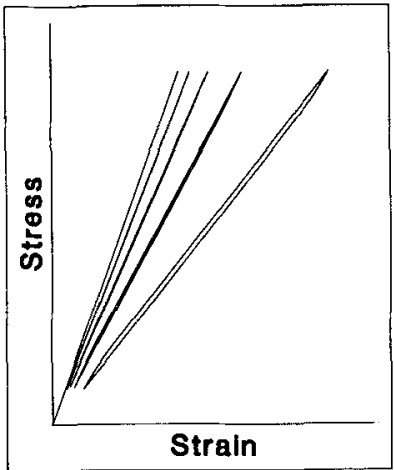

(b) $\tau_{i}=0.5 \mathrm{MPa}$

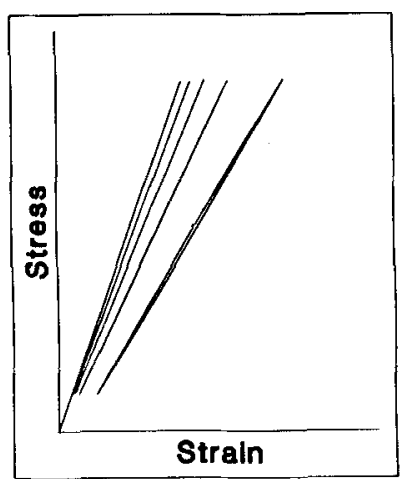

(d) $\tau_{1}=9.0 \mathrm{MPa}$
Fig. 8. Influence of interfacial friction on the predicted hysteresis in stress-strain response of $\mathrm{SiC}_{f} / \mathrm{Si}_{3} \mathrm{~N}_{4}$ composites subjected to load-controlled tensile fatigue. The stress-strain curves were calculated for a fiber volume fraction of $30 \%$; the loading conditions used in the calculations are given in Fig. 7 . From left-toright in each figure, the curves correspond to fracture of $0 \%, 20 \%$, $40 \%, 60 \%$, and $75 \%$ of all fibers. Note the lack of hysteresis predicted for both weak and strong interfacial bonding. 
mum at 2.5 MPa (Fig. 8(c)) and decreases as the frictional shear strength is increased. A maximum in strain ratchetting is predicted at $5 \mathrm{MPa}$. This peak in hysteresis at intermediate values of $\tau_{i}$ is due to the competing effects of the frictional force $F_{\text {fric }}$ and bridging of matrix cracks. Hysteresis, which is a measure of the energy dissipation per cycle, depends upon both the friction force and the length over which the fibers slide (related to the opening displacement of matrix cracks, $L_{0}$ in Fig. 2(a). The frictional force $F_{\text {fric }}$ (equal to $\pi \tau_{i} L_{e} d_{f}$ ) increases with $\tau_{i}$, whereas $L_{0}$ decreases with increasing $\tau_{i}$ because of more effective frictional bridging of matrix cracks.

(C) High Frictional Shear Strength: The predicted hysteresis becomes negligible as $\tau_{i}$ approaches $9 \mathrm{MPa}$ (Fig. 8(d)). A high frictional shear strength will tend to limit fiber pullout by locking the fractured fibers in the matrix, decreasing both hysteresis and the extent of strain ratchetting. Note also that the decreased fiber pullout length at high levels of frictional shear strength decreases the compliance of the composite (this is attributed to the decreased length over which a fiber undergoes elastic loading in the vicinity of matrix cracks).

The above analyses assumed a fiber volume fraction of $30 \%$. It is of interest to examine the influence of fiber volume fraction on the cyclic stress-strain response of the composite. With the assumption that the debond length is not influenced by volume fraction of fibers, the analysis has been performed for 15 and 45 vol\% of fibers (the results are given in Figs. 9 and 10 , respectively). For 15 vol\% of fibers, the maximum in hysteresis occurs at a $\tau_{i}$ value of $3.5 \mathrm{MPa}$; at $45 \mathrm{vol} \%$, the maximum occurs at $1.5 \mathrm{MPa}$. Thus, increasing the volume fraction of fibers shifts the maximum in hysteresis to lower values of frictional shear strength. As with 30 vol\% of fibers, hysteresis becomes negligible as the frictional shear strength approaches $9 \mathrm{MPa}$. Physically, as the volume fraction of fibers

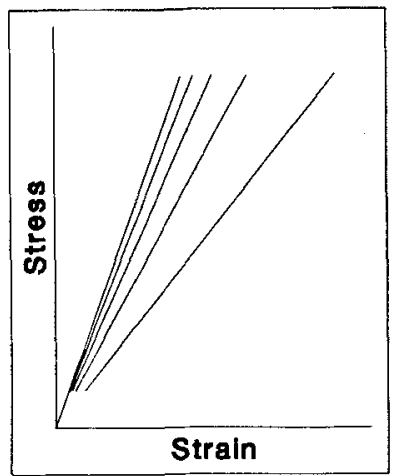

(a) $\tau_{i}=0 \mathrm{MPa}$

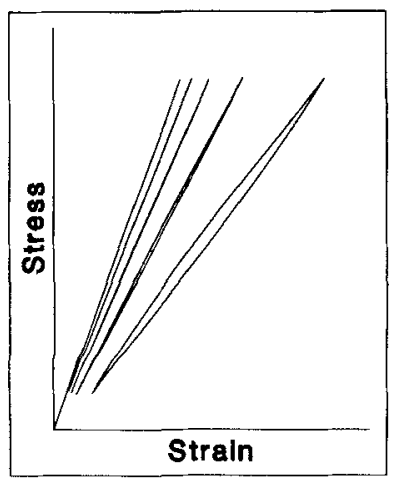

(c) $\tau_{i}=3.5 \mathrm{MPa}$

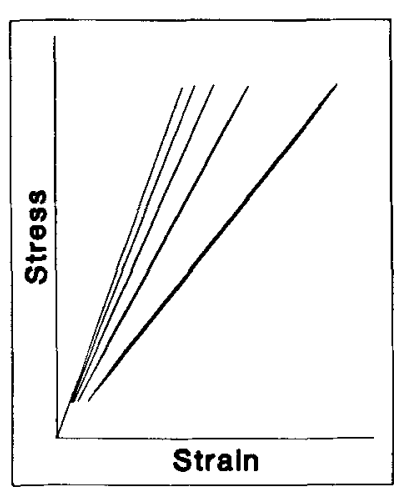

(b) $\tau_{\mathrm{i}}=0.5 \mathrm{MPa}$

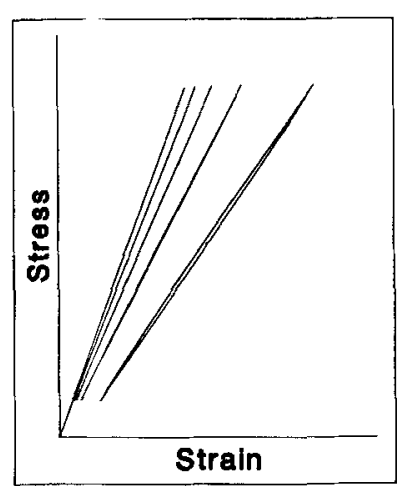

(d) $\tau_{i}=9.0 \mathrm{MPa}$
Fig. 9. Influence of interfacial friction on the predicted hysteresis in stress-strain response of unidirectional $\mathrm{SiC}_{f} / \mathrm{Si}_{3} \mathrm{~N}_{4}$ composites of low fiber fraction ( $15 \mathrm{vol} \%)$. The loading conditions used in the calculations are given in Fig. 7. From left-to-right in each figure, the curves correspond to fracture of $0 \%, 20 \%, 40 \%, 60 \%$, and $75 \%$ of all fibers.

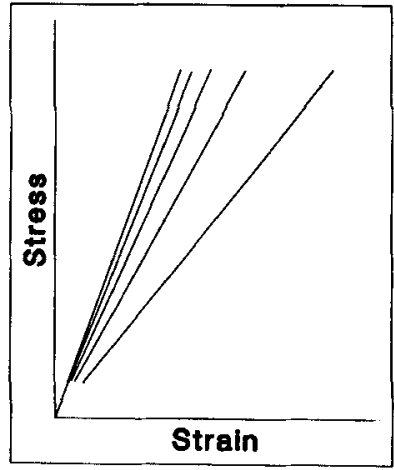

(a) $\tau_{\mathbf{i}}=0 \mathrm{MPa}$

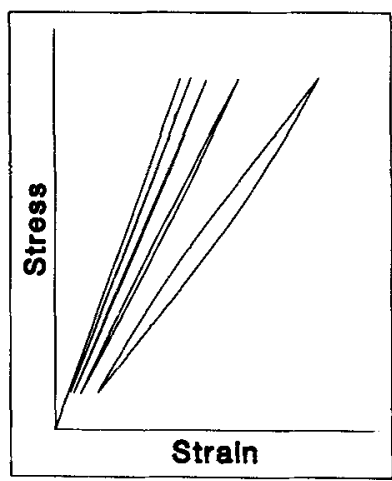

(c) $\tau_{i}=1.5 \mathrm{MPa}$

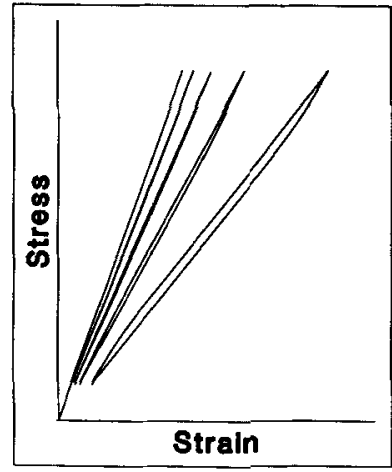

(b) $\tau_{\mathrm{i}}=0.5 \mathrm{MPa}$

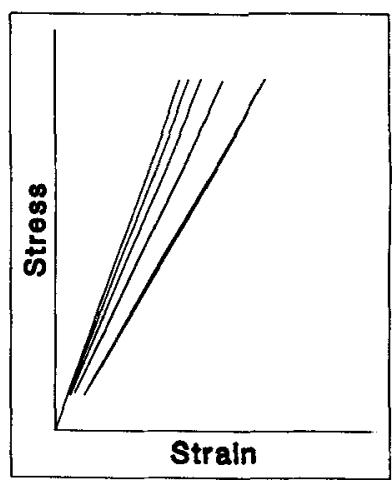

(d) $\tau_{1}=9.0 \mathrm{MPa}$
Fig. 10. Influence of interfacial friction on the predicted hysteresis in stress-strain response of unidirectional $\mathrm{SiC}_{f} / \mathrm{Si}_{3} \mathrm{~N}_{4}$ composites of high fiber fraction (45 vol\%). The loading conditions used in the calculations are given in Fig. 7. From left-to-right in each figure, the curves correspond to fracture of $0 \%, 20 \%, 40 \%$, $60 \%$, and $75 \%$ of all fibers.

increases, the total surface area available for energy dissipation increases, whereas the increase in number of fibers per unit area of matrix decreases the opening of matrix cracks through more effective frictional bridging of cracks.

The model presented here represents a first step toward understanding the influence of interfacial friction on the macroscopic fatigue response of ceramic-matrix composites. The analysis will need to be refined further to account for the variation of frictional shear with loading rate. Also, the possibility that debris from matrix and fiber fractures could partially wedge open matrix cracks during unloading needs to be considered. Finally, it should be appreciated that the present analysis has been derived for a simple unidirectional fiberreinforced ceramic at a temperature where creep deformation could be neglected. The mechanisms of fatigue damage in more complicated composites (e.g., laminated composites and composites reinforced by woven fiber strands) may differ significantly from those considered in the present analysis.

\section{Conclusions}

(1) The frictional shear strength that exists along the fiber/martix interface after initial debonding of fibers plays a significant role in determining the macroscopic fatigue response of fiber-reinforced ceramics that are subjected to fatigue loading above their proportional limit. The stressstrain hysteresis observed during fatigue is predicted to reach a maximum at moderate levels of frictional shear strength. For a given composite system, increasing the volume fraction of fibers shifts the maximum in hysteresis to lower values of frictional shear strength. Hysteresis is not predicted at very low $(<1 \mathrm{MPa})$ or high $(>9 \mathrm{MPa})$ values of frictional shear strength. 
(2) The analysis presented does not predict the "S" shape of the cyclic stress-strain curves that are sometimes observed during tensile fatigue loading of fiber-reinforced/ceramicmatrix composites. This " $\mathrm{S}$ " shape is thought to result from the variation of interfacial friction with loading rate, which has not been considered in the analysis.

\section{References}

${ }^{1}$ E.Y. Luh and A. G. Evans, "High-Temperature Mechanical Properties of a Ceramic Matrix Composite," J. Am. Ceram. Soc., 70 [7] 466-69 (1987).

${ }^{2}$ H. H. Moeller, W. G. Long, A. J. Caputo, and R. A. Lowden, "FiberReinforced Ceramic Composites"; pp. 1519-27 in Advanced Materials Technology '87. Society for the Advancement of Material and Process Engineering, Covina, CA, 1987

${ }^{3}$ W. B. Hillig, "Strength and Toughness of Ceramic Matrix Composites," Annu. Rev. Mater. Sci., 17, 341-83 (1987).

${ }^{4}$ M. D. Thouless and A. G. Evans, "Effects of Pull-Out on the Mechanical Properties of Ceramic-Matrix Composites," Acta Metall., 36 [3] 517-22 (1988).

${ }^{5}$ D. B. Marshall and B. N. Cox, "Tensile Behavior of Brittle Matrix Composites: Influence of Fiber Strength," Acta Metall, 35 [11] 2607-19 (1987).

"V. C. Nardone and K. M. Prewo, "Tensile Performance of Carbon-FibreReinforced Glass," J. Mater. Sci,, 23 [1] 168-80 (1988).

${ }^{7}$ O. Sbaizero and A. G. Evans, "Tensile and Shear Properties of Laminated Ceramic Matrix Composites," J. Am. Ceram. Soc., 69 [6] 481-86 (1986).

${ }^{8}$ R. A. Shimansky and H. T. Hahn, "Effect of Interfacial Properties on Matrix Cracking Stress of Fiber-Reinforced Ceramics"; pp. 176-82 in Symposium on High-Temperature Composites. American Society for Composites, Dayton, $\mathrm{OH}, 1989$

${ }^{9}$ D. B. Marshall and A. G. Evans, "Failure Mechanisms in Ceramic-Fiber/
Ceramic-Matrix Composites," J. Am. Ceram. Soc., 68 [5] 225-31 (1985).

${ }^{10}$ D. B. Marshall and W. C. Oliver, "Measurement of Interfacial Mechanical Properties in Fiber-Reinforced Ceramic Composites," J. Am. Ceram. Soc., 70 [8] 542-48 (1987).

${ }^{11}$ E. Minford and K.W. Prewo, "Fatigue of Silicon Carbide-Reinforced Lithium-Alumino-Silicate Glass-Ceramics"; pp. 561-70 in Tailoring Multiphase and Composite Ceramics. Edited by C. G. Patano and R. E. Messing. Plenum Press, New York, 1986.

${ }^{12}$ K. M. Prewo, "Fatigue and Stress Rupture of Silicon Carbide-FibreReinforced Glass Ceramics," J. Mater. Sci., 22, 2695-701 (1987).

${ }^{3} \mathrm{~J}$. W. Holmes, T. Kotil, and W. Foulds, "High-Temperature Fatigue of SiC-Fiber-Reinforced $\mathrm{Si}_{3} \mathrm{~N}_{4}$ Ceramic Composites”; pp. 176-82 in Symposium on High-Temperature Composites. American Society for Composites Dayton, OH, 1989.

${ }^{14}$ T. Kotil, J. W. Holmes, and M. Comninou, "Interfacial Effects in SiC Reinforced Ceramic Composites"; presented at the 3rd Joint ASCE/ASME Mechanics Conference, San Diego, CA, July 9-12, 1989.

${ }^{15}$ C. W. Goettle, D. K. Shetty, S. Y. Limaye, and D. W. Richardson, "Evaluation of Interfacial Properties in Borosilicate-SiC Composites Using PullOut Tests," Ceram. Eng. Sci. Proc., 12 [7-8] 671-78 (1988).

${ }^{16} \mathrm{~A}$. Jurewicz and R. J. Kerans, "Single-Fiber Pull-Out Test in SiC-Glass Systems"; presented at the 90th Annual Meeting of the American Ceramic Society, Cincinnati, OH, May 5, 1988 (Engineering Ceramics Division, Paper No. 155-C-88).

${ }^{17}$ R.W. Goettler and K.T, Faber, "Interfacial Shear Stresses in SiC and Alumina Fiber-Reinforced Glasses," Ceram. Eng. Sci. Proc., 9 [7-8] 861-72 (1988).

${ }^{18}$ U. V. Deshmukh and T. W. Coyle, "Determination of the Interface Strength on Glass-SiC Composites via Single-Fiber Tensile Testing," Ceram. Eng. Sci. Proc., 9 [7-8] 627-34 (1988).

${ }^{19}$ R. J. Kerans, R. S. Hay, and N. J. Pagano, "The Role of Fiber-Matrix Interface in Ceramic Composites," Am. Ceram. Soc. Bull., 68 [2] 429-42 (1989). 